\title{
Carnets
}

Revue électronique d'études françaises de l'APEF

Deuxième série - $10 \mid 2017$

Pratiques de l'errance, vécus de la mémoire

\section{La shoah post-mémorée à la troisième génération}

L'exemple de Marianne Rubinstein : essai et récit

José Domingues de Almeida

\section{(2) OpenEdition}

Journals

Édition électronique

URL : http://journals.openedition.org/carnets/2209

DOI : $10.4000 /$ carnets.2209

ISSN : 1646-7698

Éditeur

APEF

Référence électronique

José Domingues de Almeida, «La shoah post-mémorée à la troisième génération », Carnets [En ligne], Deuxième série - 10 | 2017, mis en ligne le 30 avril 2017, consulté le 01 mai 2019. URL : http:// journals.openedition.org/carnets/2209; DOI : 10.4000/carnets.2209

Ce document a été généré automatiquement le 1 mai 2019.

\section{(c) (i) \&}

Carnets est mis à disposition selon les termes de la licence Creative Commons - Atribution - Pas d'utilisation commerciale 4.0 International. 


\title{
La shoah post-mémorée à la troisième génération
}

\author{
L'exemple de Marianne Rubinstein : essai et récit
}

José Domingues de Almeida

Ils s'aimaient. Mon père s'en souvient, et je le sens

à la lumière de ce que j'ai lu, ou entendu

C'est maintenant du passé, p. 13

Ce fils d'Abraham Rubinstein, ce cousin germain de mon père qui devait avoir douze ans en 1942, je ne

connais même pas son prénom. Que cela ne m'empêche pas de me souvenir

idem, p.66

1 La démarche narrative post-mémorielle de Marianne Rubinstein sur la scène littéraire française contemporaine ne constitue nullement un cas isolé1. Il suffit pour s'en convaincre de consulter la liste de titres récents - certains primés, dont Les Bienveillantes de Jonathan Littell (2006) - qu'en donne Évelyne Ledoux-Beaugrand, à quoi s'ajoute l'émergence d'une expression littéraire juive non spécifiquement redevable au travail post-mémoriel de la Shoah, mais qui s'y réfère autrement, par la mise en jeu de repères historiques et culturels immédiatement identifiables, comme P. Claudel, A. Rykner, K. Tuil, O. Benyahya, ou L. Binet) qui, eux aussi, relaient et revisitent en quelque sorte dans la fiction le trauma de la Shoah enduré par leurs aïeuls durant la Seconde Guerre mondiale (déportations, morts en masse, résistances, souvenirs de guerre, humiliations diverses, etc.).

Stéphanie Bellemare-Page insiste :

L'époque actuelle se caractérise par la multiplication des espaces consacrés à la mémoire individuelle et collective. On ne compte plus les monuments, les musées, les discours destinés à nous rappeler certains épisodes-clés de notre histoire, notamment celle du siècle dernier. Ce phénomène est d'autant plus important que nous avons désormais la capacité, d'un point de vue technique, d'accumuler des quantités phénoménales de documents de toutes sortes (2006:49). 


\section{travail narratif de Marianne Rubinstein. Cette notion - communément attribuée aux}

travis, creusons d’abord le concept de "post-mémoire " par lequel nous caractérisons le travaux et à l'expérience personnelle de Marianne Hirsch - désigne très précisément :

(...) la relation que la " génération d'après " entretient avec le traumatisme personnel, collectif et culturel subi par ceux qui l'ont précédée, avec des expériences dont elle ne « se souvient » que par le biais d'histoires, d'images et de comportements au milieu desquels elle a grandi. Mais ces expériences lui ont été transmises si profondément et avec tant d'émotion qu'elles semblent constituer une mémoire en tant que telle. Comme [je] la conçois, la connexion avec le passé que je définis comme postmémoire ne s'opère pas au travers d'une forme particulière de remémoration, mais d'un investissement imaginaire, d'une projection et d'une création (Hirsch, $2008: 114$ ).

4 Il s'agit dès lors, selon elle, $d^{\prime}$ '« une forme indirecte de mémoire $»^{2}$, qui, de fait, comme l'attestent la tenue de plus d'un colloque, est devenu simultanément « un important outil d'analyse et un objet d'étude (...)» (ibidem).

Dans le domaine français, mais pas uniquement, cette notion paradoxale du fait du préfixe - tout comme le fut celle de " postmodernité » (Vattimo, 1987) dans les années quatre-vingt - n'a pas manqué de susciter des réticences définitoires. Comme le rappelle Évelyne Ledoux-Beaugrand, « entre la mémoire et la postmémoire, il ne s'agirait pas seulement d'une différence de degrés dans la connexion aux événements du passé, ni d'une simple distanciation temporelle, mais d'une différence fondamentale qui devrait se marquer, selon Van Alphen, par l'utilisation d'un terme autre » (2013: 148), ce qui requiert des précisions de nomenclature.

6 Ledoux-Beaugrand nuance l'acception du mot. Selon elle, « En dépit de son nom, la postmémoire ne désigne pas un processus mémoriel au sens courant de la réminiscence d'événements dont un sujet a fait l'expérience. L'équivoque autour de cette notion tient à ce qu'elle peut laisser penser à la possibilité de se remémorer des événements vécus par d'autres » (ibidem) vu que, dans ce cas précis,

(...) le vocable mémoire ne renvoie pas à la faculté cognitive d'enregistrer, de préserver et de restituer les souvenirs d'expériences vécues. Il signale plutôt une connexion au passé établie par une forme d'appropriation créative qui s'efforce de réactiver et de réincorporer des structures mémorielles distantes (idem : 149).

7 Dans un essai qui a connu un franc succès, La Mémoire saturée (2003), Régine Robin caractérisait la post-mémoire comme la «transmission de traumatismes de la guerre ou du génocide par ceux qui n'ont pas connu la guerre ou qui étaient trop jeunes pour comprendre la gravité des événements » (idem: 322 ), c'est-à-dire, comme le reprend et précise Stéphanie Bellemare-Page, que « (...) le concept de postmémoire évoque plus particulièrement la démarche créatrice des enfants de victimes de la Shoah qui, par l'entremise de l'art ou de l'écriture, parviennent aujourd'hui à exprimer, à leur manière, leur souvenir des récits que leur ont fait leurs parents » (2006:50), cette mémoire qualifiée d'« indirecte » ne pouvant se constituer que dans une dimension imaginaire ( ibidem).

$8 \mathrm{Si}$, au départ, cette notion entretenait avec la Shoah juive un rapport référentiel quasi exclusif, notamment en vertu du caractère effroyable du génocide perpétré en Europe malgré les métarécits modernes et l'humanisme dans ses multiples facettes religieuses et philosophiques, mais aussi du fait du statut victimaire collectif particulier qui la question juive a suscité dans la conscience occidentale (Finkielkraut, $2007: 26$ ), elle se voit également appliqué pour le vécu mémoriel médiat motivé par d'autres tragédies 
historiques : Rwanda, ex-Yougoslavie, Cambodge, Indiens, Aborigènes, Noirs déportés en esclavage, etc.

Toutefois, pour ce qui de la réminiscence vécue par la question juive où la Shoah condense et résume la tragédie, l'horreur, la collaboration, la mauvaise conscience collective, la souffrance, le projet d'extermination et le souvenir, là, le travail postmémoriel assume un statut particulier de par la quantité, la qualité et la proximité documentaires, testimoniales, voire revendicatives. Giorgio Agamben a bien caractérisé la problématique du « reste » d'Auschwitz comme matière plurielle et aux multiples facettes - dont les formes narrativisées et les discours mémoriels - offerte à la post-mémoire, d'autant plus que le caractère palliatif du témoignage fondé sur une parole vivante s'effiloche au fur et à mesure que ces témoins expérientiels disparaissent, et qu'en fait le seul témoignage qui eût compté n'est autre que celui des gazés (1999 : 31-48), ce qui inaugure une ère post-mémorielle privée de témoins à laquelle prélude l'étude d'Annette Wieviorka dans L'Ère du témoin (1998).

Dans son étude critique sur Marianne Rubinstein et Yannick Haenel, Évelyne LedouxBeaugrand précise l'acception de l'écriture post-mémorielle qu'elle détecte chez ces deux auteurs chez qui le souvenir de la Shoah ne peut engendrer qu'une expérience distancée et médiate. Selon elle, le travail narratif post-mémoriel « (...) consiste à adopter un point de vue ancré dans le présent et à ainsi aborder le génocide à partir de ses traces encore repérables dans l'espace et le temps contemporains. [II] s'emploie moins à jeter un regard sur le passé qu'à tirer au jour les restes de ce passé dont est pétri le présent » (2013: 146).

Deux textes narratifs intimement liés du point de vue thématique de Marianne Rubinstein retiendront notre attention : une enquête-documentaire et un récit, respectivement Tout le monde n'a pas la chance d'être orphelin (2002) - dont Serge Klarsfeld, militant majeur de la mémoire de la Shoah - assure la préface (idem : 7-13), et C'est maintenant du passé (2009). Ces deux textes ont en commun le souci d'un rendu post-mémoriel de l'expérience de la Shoah à partir du regard d'enfants d'orphelins de parents juifs français déportés et exterminés dans les camps de concentration allemands lors de la Deuxième Guerre mondiale, dont Marianne Rubinstein elle-même.

12 Si dans le premier texte, symptomatiquement dédicacé au père et au fils de Marianne Rubinstein, il s'agit surtout de sonder comparativement, sous forme d'enquête et par le biais d'une suite de portraits, l'expérience et le vécu intimes de la Shoah et de la condition juive éprouvés par la troisième génération, dans le deuxième, on a affaire à une écriture " façonné[e] par [1]a posture d'héritière d'un récit familial sur la Shoah et ses conséquences » (Ledoux-Beaugrand, 2013 : 147).

13 Il s'en dégage des constantes ou des récurrences post-mémorielles pertinentes pour notre étude à partir du recueil des témoignages d'Élise, Marc-Olivier, Frédéric, Catherine, Jacques, Michael, Aude, Sylvie, Claire, Laurent, David, Gérard, Guillaume, François, Reine et Marianne Rubinstein elle-même (témoignage en italique), tous «témoins » postmémoriels de la Shoah marqués par l'état d'orphelinat de leurs parents. Comme le rappelait Marianne Hirsch, si :

La deuxième génération s'est comportée en gardien, déterminant les formes de transmission qui étaient valables et celles qui ne l'étaient pas (...) il nous faut maintenant prendre conscience que nous devons nous-mêmes transmettre l'histoire à la troisième génération et la mettre à la disposition d'autres personnes, de telle sorte qu'elles puissent la relier à leurs propres histoires, qui sont très diverses (ibidem) 

notamment par ses représentations littéraires » (Ledoux-Beaugrand, 2013 : 146) ; ou ses représentations tout court, mais aussi ses séquelles subtiles distanciées qui se traduisent par des topoï post-mémoriels récurrents. Relevons-en les plus importants. Et tout d'abord, la tendance généralisée à une reviviscence très libre et soudaine des traditions juives en vue d'un regain identitaire positif (Rubinstein, 2002: 109, 112) afin de dépasser l'exclusivité du rapport familial à la Shoah comme seule marque d'appartenance juive, un fait souvent inspiré de la lecture de l'essai prégnant Le Juif imaginaire d'Alain Finkielkraut (1981). Élise avouera que « Le rapport à la Shoah et le drame familial de mon père [orphelin] ont toujours été la seule relation au judaïsme et à la judéité » (Rubinstein, $2002: 25,35,61,94)$. difficulté à se dire Français après ce qui s'est passé et a affecté leurs aïeuls (idem: 92), ce qui revient à démonter les clichés d'une France patrie des droits de l'homme et terre d'accueil (idem: 62). Mais aussi une peur multiforme, enfouie et latente : peur de l'abandon ou de ne laisser aucune descendance (idem : 36-37, 104), panique devant l'idée de la perte (idem: 72-73), peur que tout ne se reproduise (idem : 97) et de mourir (idem : 99). inexprimé des affres vécues par les grands-parents lors de la Shoah qui s'avèrent indiciels et symptomatiques d'un héritage déphasé dans le temps. Remarquons l'assomption d'un devoir moral transmis : transfert des ambitions des aïeuls (jouer du violon, du piano) ( idem : 47), expérience d'une mère obsédée par le rangement (idem : 103), enfance gâtée pour compenser l'horreur héritée d'un passé encore récent (idem : 48), fascination pour les tatouages (idem: 102), mais aussi le sentiment de culpabilité d'avoir été historiquement épargné de l'extermination. David exprime ce sentiment douloureux aux prises avec la post-mémoire :

« (...) J'ai plutôt l'impression que je n'arrive pas à faire le deuil. [Dans ma famille], on occulte la souffrance, on ne parle pas des choses qui ne vont pas, on avance, la vie continue » (...). « Ce que je ressens peut-être, c'est le regret de ne pas avoir vécu cette période, presque de la culpabilité. J'aurais dû y être pour me battre; non pas pour partager [la mort mais] pour refaire l'histoire » (idem : 120).

On le voit: la troisième génération de la Shoah accuse à présent le coup du trauma collectif horriblement subi par ses aïeuls et, forcément, par les nombreux orphelins des camps d'extermination que furent ses parents. En préface à l'enquête de Marianne Rubinstein, Serge Klarsfeld en appelle à la mission et au statut assignés aux enfants des orphelins de la Shoah et souligne l'importance de ce travail inédit. Selon lui, « Il révèle comment les traumatismes se sont perpétués dans la descendance des survivants des familles » (idem : 11). Quel sera le rôle de la quatrième génération ? L'auteur ose espérer qu'il sera plutôt pacificateur : « (...) la naissance de mon fils m'[a] aidée à pacifier ma relation à ma propre histoire » (idem : 124).

Cette enquête, Marianne Rubinstein a fini par la porter sur sa propre expérience postmémorielle de fille d'un orphelin français de la Shoah dans un récit qui, du fait même de son propos testimonial, évite la saga familial (Rubinstein, 2009 : 38-39) et se sépare d'avec le genre romanesque (idem: 40,117). Rappelons que si le titre du récit n'est que la traduction de la bordure narrative japonaise " Il était une fois », c'est le fragment 
testimonial qui est ici rendu et c'est le fragment qui est visé, comme le suggère l'intermittence du haïku (idem : 79).

Dans son approche de la post-mémoire, Marianne Hirsch évoque «(...) des fragments traumatiques d'événements qui continuent à défier la reconstruction narrative et à excéder la compréhension. Ces événements se sont produits dans le passé, mais leurs effets se prolongent dans le présent » (ibidem), ce que Stéphanie Bellemare-Page désigne par « strate mémorielle » $(2006: 52)$.

Rubinstein entend d'emblée afficher ce caractère inachevé et inachevable de son projet, ce qui le renvoie au labeur de reconstitution du patchwork (Rubinstein, 2009: 154), du lambeau de vie (idem : 137, 150), du puzzle (idem: 133), et partant du fragment, c'est-àdire le salut de vies perdues; ce que traduit le recours modal au conditionnel dans son rapport post-mémoriel aux grands-parents déportés et exterminés : «Je me dis que je les aurais aimés, que j'aurais eu ma place auprès d'eux; qu'il aurait eu des yeux pour voir; qu'elle m'aurait aidée à accepter ma part d'inacceptable " (idem: 17). À cet égard, l'absence et la recherche (idem : 142) obsessionnelle d'une « place » dans l'histoire, sur les photos, dans la mémoire des autres ou dans la filiation ponctue le récit (idem : 25, 26, 127).

21 Ceci dit, nous avons bel et bien affaire ici à ce que Marianne Hirsch nomme " la postmémoire familiale " pour la distinguer de " la postmémoire affiliative », qui n'est pas éprouvée par un descendant direct du traumatisme passé (ibidem). Aussi, « le récit de Rubinstein est[-il] façonné par sa posture d'héritière d'un récit familial sur la Shoah et ses conséquences » (Ledoux-Beaugrand, $2013: 147$ ).

Deux éléments et événements fondateurs déclenchent et balisent cette quête personnelle post-mémorielle. D'une part, il y a le trauma familial et la césure de l'arrestation des grands-parents de Rubinstein à la gare de Montchanin et la douloureuse et définitive séparation d'avec Serge, le père de Marianne, orphelin, confié aux soins de la famille Szpirglas :

Il [le père] se souvient de cet anniversaire. Après la rafle du Vél'd'Hiv du 16 juillet 1942, il prend le train avec ses parents en direction de la zone libre. Peut-être rêve$\mathrm{t}$-il du spectacle au cirque d'Hiver, où on l'emmènera, c'est promis, lorsque la guerre sera finie. À Montchanin, en Saône-et-Loire, au passage de la ligne de démarcation, il y a une rafle sur le quai. Quand ils finissent par descendre, il n'y a plus personne. La gare est vide, mais au moment où ils franchissent la porte pour se retrouver dans la rue, un officier allemand les arrête. "Et l'histoire s'arrête là " (Rubinstein, 2009: 102).

D'autre part, il y a la décision d'ouvrir la boîte aux souvenirs du père, celle qui contient la correspondance épistolaire échangée entre plusieurs membres de la famille lors de la déportation, dont celles du grand-père, mais aussi des photos prises lors de plusieurs occasions festives. Ce moment, sans cesse différé, devient l'occasion pour Marianne Rubinstein de sonder et de tenter de reconstituer un tracé identitaire et familial fondé sur des pièces isolées qu'il s'agit de faire parler. S'enfoncer dans cette boîte revient métaphoriquement à quête régressive dans le passé :

La "boîte aux souvenirs " de mon père est une modeste boîte en fer bleue. Nous l'ouvrîmes le mardi 13 novembre 2007. Je me souviens mal de ce que nous y trouvâmes ce jour-là. Au vu de mes notes, surtout des factures, des dates, des adresses, des papiers administratifs. La boîte semblait profonde, et nous avancions lentement (idem : 34 ).

Selon Aurélie Barjonet, chez Marianne Rubinstein «L'histoire familiale donne toujours lieu à un texte archéologique ", alors que "la majorité des écrivains s'est donc tournée 
vers la transmission ou la fictionnalisation d'une histoire vraie » (2012: 41). De ce fait, nous sommes en présence d'un récit post-mémoriel où, d'après Évelyne LedouxBeaugrand, « la postmémoire nomme à la fois une structure de transmission trans et intergénérationelle et la posture d'une génération face à des événements traumatiques éloignés d'elle dans le temps et parfois aussi dans l'espace et dans la langue » (2013: 148). Rubinstein l'affirme autrement: "Sans doute voulais-je mieux cerner l'emprise d'une histoire que nous n'avions pas vécue, et qui pourtant pesait sur nos vies " (Rubinstein, 2009 : 29), et pointe inconsciemment l'essence même de la posture post-mémorielle : " N'est-ce pas aussi le cas pour les traumatismes survenus après la guerre ? Que valent-ils à côté de la destruction massive d'un monde ? (idem : 57).

On l'aura compris : la place des documents familiaux et de la recherche de témoignages s'avère cruciale dans la démarche post-mémorielle. Marianne Hirsch souligne le rôle des archives digitales (...) illimitées, sans lieu, mobiles. Je vois l'internet et le web comme des espaces dans lesquels la mémoire circule grâce à la possibilité de se connecter et je pense qu'il est urgent que nous réfléchissions à ces nouvelles dimensions de la mémoire, qui jouent sur la comparaison et la connexion (ibidem).

Évelyne Ledoux-Beaugrand se veut, à ce titre, catégorique : « (...) le recours à des sources intimes et familiales (photographies, lettres, journaux intimes, correspondances, histoires de famille transmises par bribes) relève généralement de la nécessité dans les œuvres postmémorielles qui cherchent à réactiver la mémoire d'anonymes disparus dans les camps nazis » (2013: 149), d'autant plus que «(...) le travail de la postmémoire donne prévalence à des sources éminemment subjectives, sans toutefois renier complètement les sources historiques » (ibidem).

La consultation de ces archives éveille des processus de transfert et de projection dans le passé familial et ses acteurs. Rubinstein souligne des rapprochements qu'elle pressent au fur et à mesure qu'elle reconstitue l'histoire familiale, et notamment des ressemblances de caractère : " J'aime excessivement la courtoisie (...). À voir les photos, mon grand-père devait être un homme courtois, le doigt droit sur le menton haut » (Rubinstein, 2009 : 17). De même, une ressemblance physique avec la grand-mère s'impose au vu des photos de famille et des témoignages des proches, descendants des membres de la famille déportés : « (...) mon père a eu ce raisonnement alambiqué qui m’a mis les larmes aux yeux : 'La meilleure preuve que je suis bien le fils de ma mère, c'est toi. Mes cousines me l'ont dit. Elles m'ont dit : 'C'est incroyable comme Marianne ressemble à ta mère' » (idem : 71-72, cf. aussi 128). Lui ressembler, c'est quelque part vouloir la rejoindre dans son destin tragique, le revivre autrement, et a posteriori se retrouver, assumer une identité, trouver une place dans l'histoire traumatique : «Tous, nous fûmes frappés par ma ressemblance avec cette grand-mère morte parce qu'elle était 'trop juive', comme je le disais vers troisquatre ans, une époque où il m'arrivait de pleurer, accrochée à mon lit et répétant sans cesse : 'Je ne veux pas mourir, je ne veux pas mourir' » (idem : 109).

Si, pour la deuxième génération de la Shoah, la dépression avait été profonde - comme celle vécue par le père de Marianne qui, à l'âge de quarante-six ans, âge du décès de son propre père, ne supportait pas de "dépasser l'âge de son père ", et avait souvent engendré un désir de justice et de réparation incarné par la militance de Juifs tels que Klarsfeld - pour la troisième génération, " ce processus témoigne (alors) d'une forme d'extériorisation curative (...) » (Bellemare-Page, 2006: 55). Stéphanie Bellemare-Page y voit " l'exercice est particulièrement intéressant chez les artistes et auteurs contemporains pour qui l'art occupe une fonction de résilience » (ibidem). Tout comme 
celle d'Andreï Makine, le travail narratif de Marianne Rubinstein atteste du rapport postmémoriel de l'écriture littéraire à la résilience » (ibidem).

Marianne Rubinstein rend à merveille cette mission et ce pouvoir de l'écriture: " Pourquoi écrire? 'Bon qu'à ça', répondait Beckett. Comment être plus laconique ? J'écris parce que j'ai un problème de place (...). J'écris parce que c'est le seul endroit d'où je peux, sans l'aide de personne, calmer l'angoisse » (Rubinstein, $2009: 22$ ).

\section{BIBLIOGRAPHIE}

AGAMBEN, Giorgio (1999). Ce qui reste d'Auschwitz. L'archive et le témoin. Homo sacer III. Paris : Payot \& Rivages.

BARJONET, Aurélie (2012). « La troisième génération devant la Seconde Guerre mondiale : une situation inédite », Études Romanes de Brno, vol. 33, nํ1, pp. 39-55.

BELLEMARE-PAGE, Stéphanie (2006). « La littérature au temps de la post-mémoire : écriture et résilience chez Andreï Makine », Études littéraires, vol. 38, n 1, pp. 49-56.

FINKIELKRAUT, Alain (1981). Le Juif imaginaire. Paris : Seuil.

FINKIELKRAUT, Alain (2007). Qu'est-ce que la France?. Paris : Stock / Panama.

HIRSCH, Marianne (2008). « The generation of postmemory », Poetics Today, vol. 29, no 1, pp.

103-128.

LEDOUX-BEAUGRAND, Evelyne (2013). « Les restes d'Auschwitz : intertextualité et postmémoire dans Jan Karski de Yannick Haenel et C'est maintenant du passé de Marianne Rubinstein », Études françaises, vol. 49, $\mathrm{n}^{\circ} 2$, pp. 145-162.

LITTELL, Jonathan (2006). Les Bienveillantes. Paris : Gallimard.

ROBIN, Régine (2003). La Mémoire saturée. Paris : Stock.

RUBINSTEIN, Marianne (2002). Tout le monde n'a pas la chance d'être orphelin. Paris : Verticales.

RUBINSTEIN, Marianne (2009). C'est maintenant du passé. Paris : Verticales.

VATTIMO, Gianni (1987). La Fin de la modernité : nihilisme et herméneutique dans la culture post-

moderne. Paris : Seuil, coll. « L'ordre philosophique ».

WIEVIORKA, Annette (1998). L’Ère du témoin. Paris : Hachette.

http://www.ciremm.org/wp-content/uploads/2015/06/Pages-de-ArtAbsPostmemoire-72dpi.pdf

\section{NOTES}

1. Cet article s'insère dans la recherche menée au sein du Programme Stratégique intégré UID/ ELT/00500/2013 | POCI-01-0145-FEDER-007339. 


\section{RÉSUMÉS}

Il s'agira de caractériser, d'après le concept opératoire de " post-mémoire » mis en orbite par Marianne Hirsch, la pensée essayistique et la fiction narrative de l'écrivaine française d'origine juive Marianne Rubinstein, plus précisément Tout le monde n'a pas la chance d'être orphelin (2002) et C'est maintenant du passé (2009). En fait, Marianne Rubinstein s'inscrit dans le groupe croissant d'écrivains de langue française (tels J. Littell, P. Claudel, Y. Haenel, A. Rykner, K. Tuil, O. Benyahya, F. Humbert, T. Hesse, ou L. Binet) qui relayent et revisitent quelque part dans la fiction le trauma de la Shoah enduré par leurs aïeuls durant la Seconde Guerre mondiale (déportations, morts en masse, résistances, souvenirs de guerre, humiliations diverses, etc.). À cet égard, comme le rappelle pertinemment Aurélie Barjonet à propos de Marianne Rubinstein, «L'histoire familiale donne toujours lieu à un texte archéologique ", alors que "La majorité des écrivains s'est donc tournée vers la transmission ou la fictionnalisation d'une histoire vraie ". C'est au croisement de ces deux axes (autobiographie et fiction) que nous tâcherons de placer l'écriture de Marianne Rubinstein.

We will characterize the essayistic thought and the narrative fiction of French writer of Jewish origin, Marianne Rubinstein, according to the operative concept of " post-memory " put into orbit by Marianne Hirsch, more precisely her novels Tout le monde n'a pas la chance d'être orphelin (2002) and C'est maintenant du passé (2009). In fact, Marianne Rubinstein belongs to the growing group of French writers (such as J. Littell, P. Claudel, Y. Haenel, A. Rykner, K. Tuil, O. Benyahya, F. Humbert, T. Hesse, L. Binet) who relay and revisit somehow in fiction the trauma of the Holocaust endured by their ancestors during the Second World War (deportations, mass deaths, resistance, memories of war, various humiliations, etc.). In this respect, as Aurélie Barjonet has rightly pointed out about Marianne Rubinstein, "Family history always gives rise to an archaeological text ", while " the majority of writers have turned to the transmission or fictionalization of 'a true story'». It is at the crossroads of these two axes (autobiography and fiction) that we will try to place Marianne Rubinstein's writing.

\section{INDEX}

Keywords : post-memory, Rubinstein (Marianne), Shoah, novel

Mots-clés : post-mémoire, Rubinstein (Marianne), Shoah, roman

\section{AUTEUR}

\section{JOSÉ DOMINGUES DE ALMEIDA}

Un. Porto - APEF - ILC ML

jalmeida[at]letras.up.pt 